\title{
Stability modulo singular sets
}

by

\author{
J. Iglesias, A. Portela and A. Rovella (Montevideo)
}

\begin{abstract}
A new concept of stability, closely related to that of structural stability, is introduced and applied to the study of $C^{1}$ endomorphisms with singularities. A map that is stable in this sense is conjugate to each perturbation that is equivalent to it in a geometric sense. It is shown that this kind of stability implies Axiom A and $\Omega$-stability, and that every critical point is wandering. A partial converse is also shown, providing new examples of $C^{3}$ structurally stable maps.
\end{abstract}

1. Introduction. Denote by $C^{r}(M)$ the space of $C^{r}$ self-mappings of a manifold $M, 1 \leq r \leq \infty$. If $M$ is compact this space has the usual $C^{r}$ topology, while for noncompact $M$ it is endowed with the Whitney (or strong) topology.

Given $f \in C^{r}(M)$ the set of critical (or singular) points of $f$ (denoted $S_{f}$ ) is the set of points where the differential of $f$ is singular.

Two maps $f$ and $g$ of class $C^{1}$ are called geometrically equivalent if there exist $C^{1}$ diffeomorphisms $\varphi$ and $\psi$ of $M$ such that $\varphi f=g \psi$. In this case, the image of a critical point of $f$ under $\psi$ is a critical point of $g$, and the image of a critical value of $f$ under $\varphi$ is a critical value of $g$. A map is called $C^{r}$ geometrically stable if there exists a $C^{r}$ neighborhood $\mathcal{U}$ of $f$ such that every $g \in \mathcal{U}$ is geometrically equivalent to $f$. Moreover, for each neighborhood $\mathcal{Z}$ of the identity of $M$ in $C^{0}$ topology, the maps $f$ and $g$ are said to be $\mathcal{Z}$-geometrically equivalent if the diffeomorphisms $\varphi$ and $\psi$ are contained in $\mathcal{Z}$. Any two $C^{1}$ diffeomorphisms are geometrically equivalent, and on compact manifolds, two endomorphisms without critical points are geometrically equivalent if and only if the absolute values of their degrees are equal. But the concept is purely geometric, it has no dynamical meaning: for example, two quadratic polynomials of one variable are always geometrically equivalent.

2000 Mathematics Subject Classification: Primary 37C20; Secondary 58K05.

Key words and phrases: structural stability, critical points of maps. 
In this article, the notion of stability of maps is considered. Two maps $f$ and $g$ are topologically equivalent if there exists a homeomorphism $h$ such that $f h=h g$. A $C^{r}$ map $f$ is $C^{r}$ structurally stable if there exists a $C^{r}$ neighborhood $\mathcal{U}$ of $f$ such that $f$ is topologically equivalent to each $g \in \mathcal{U}$.

In [IPR1], a concept of stability of maps was introduced that generalizes the usual concept and is more adequate to study maps having critical points.

Definition 1. A map $f \in C^{r}(M)$ is called $C^{r}$ structurally stable modulo singular sets, denoted $f \in I^{r}(M)$, if there exist a neighborhood $\mathcal{Z}$ of the identity in $C^{0}(M)$ and a $C^{r}$ neighborhood $\mathcal{U}$ of $f$ such that two $\mathcal{Z}$ geometrically equivalent maps $g_{1}$ and $g_{2}$ in $\mathcal{U}$ are topologically equivalent.

An obvious observation, which can be used to find structurally stable maps, is the following:

If $f$ is $C^{r}$ geometrically stable and belongs to $I^{1}(M)$, then $f$ is $C^{r}$ structurally stable.

The need of $\mathcal{Z}$-geometric equivalence (instead of geometric equivalence) will become apparent in Theorem C. If $f$ is $C^{r}$ structurally stable then it belongs to $I^{r}(M)$. Under generic assumptions on the maps $g_{1}$ and $g_{2}$, topological equivalence implies geometric equivalence.

A map having critical points cannot be $C^{1}$ structurally stable, but a map $f \in I^{1}(M)$ has the following property: given a $C^{0}$ neighborhood $\mathcal{Z}$ of the identity, there exists a $C^{1}$ neighborhood $\mathcal{U}$ of $f$ such that $\mathcal{Z}$-geometric equivalence is an equivalence relation in $\mathcal{U}$ and coincides with topological equivalence in $\mathcal{U}$. Lemma 3 at the end of Section 2 implies that if a map $f$ belongs to $I^{1}(M)$, then $f$ is topologically equivalent to any $C^{1}$ perturbation $g$ that coincides with $f$ in a neighborhood of $S_{f}$.

Necessary and sufficient conditions for a diffeomorphism $f$ to be $C^{1}$ structurally stable are well known (Robinson, [R], 1976 and Mañé [Ma1], 1987). Since then, no new examples of $C^{r}(r \geq 2)$ structurally stable diffeomorphisms have been discovered; the question whether there exist any remains open. Other $C^{r}$ stable maps are known: stability of expanding maps in compact manifolds was proved by Shub $[\mathrm{S}]$. The case of one-dimensional maps, where the situation is easier, will not be discussed here.

New examples (apart from the diffeomorphism or expanding cases) of $C^{1}$ structurally stable maps were provided in [IPR2]. Berger [Ber] has recently found sufficient conditions and examples of $C^{\infty}$ structurally stable maps.

The first known examples of $C^{3}$ (but not $C^{2}$ ) stable maps were constructed in [IPR1]. In that article, it was first proved that a rational map of the Riemann sphere with the no critical relations property (i.e., no critical point belongs to the forward orbit of another critical point and no critical point is periodic) and whose Julia set is hyperbolic and connected, belongs 
to $I^{1}\left(\mathbb{R}^{2}\right)$. Then these maps were approximated by $C^{3}$ geometrically stable maps, providing the $C^{3}$ structural stability.

Theorems A and B describe some properties of $I^{1}(M)$ maps.

Theorem A. Let $M$ be a compact manifold. If $f \in I^{1}(M)$, then every critical point of $f$ is wandering, and $f$ is Axiom $A$ and $C^{1} \Omega$-stable.

The definitions involved are the following: a point is wandering if it has a neighborhood $U$ such that $f^{n}(U) \cap U=\emptyset$ for every $n>0$. The set of nonwandering points of $f$ is denoted by $\Omega(f)$. Two maps $f$ and $g$ are $\Omega$-equivalent if there exists a homeomorphism $h: \Omega(f) \rightarrow \Omega(g)$ such that $h f=g h$ in $\Omega(f)$. A map $f$ is $C^{r} \Omega$-stable whenever all its $C^{r}$ perturbations are $\Omega$-equivalent to it.

A map $f$ whose nonwandering set has a hyperbolic structure and whose set of periodic points is dense in $\Omega(f)$, has a spectral decomposition: the nonwandering set of $f$ is the union of a finite number of basic pieces; these are compact, invariant, transitive sets. Then the map is called Axiom $A$ if, in addition, the restriction of $f$ to each basic piece is either injective or expanding. (This is the definition given in [Ma2]; other authors call this concept strong Axiom $A$; and Przytycki $[\mathrm{P}]$ does not require this last condition for a map to be Axiom A). A basic piece $\Lambda$ is expanding if the stable subspace at $x, E_{x}^{\mathrm{s}}$, is $\{0\}$, for $x \in \Lambda$. A basic piece $\Lambda$ is called a repeller if the stable manifold of each point in $\Lambda$ is contained in $\Lambda$. In this case, the unstable set of $\Lambda$, denoted $W^{\mathrm{u}}(\Lambda)$, and defined as the set of points $x \in M$ having a preorbit whose limit set is contained in $\Lambda$, is a neighborhood of $\Lambda$. Not every repeller is expanding, but if the map is Axiom A, then every repeller that is not expanding must be injective.

Another necessary condition for a map $f$ to belong to $I^{1}(M)$ is that the critical set must be contained in a particular region of the wandering set:

Theorem B. If $f \in I^{1}(M)$ and $C$ is a component of the critical set $S_{f}$ of $f$, then:

- There exists an attracting periodic orbit $\gamma$ such that $C$ is contained in its basin of attraction.

- If $C$ intersects the unstable set of a basic piece $\Lambda$, then $\Lambda$ is expanding and $C$ is contained in its unstable set.

It remains an open problem whether maps in $I^{r}(M)$ for $r>1$ have to satisfy any of the conclusions of Theorems A and B. No examples of maps in $I^{r}(M) \backslash I^{1}(M)$ with $r>1$ are known.

There is another necessary condition for a map to belong to $I^{1}(M)$. This condition was found necessary for $C^{1}$ structural stability by Przytycki (Theorem $\mathrm{C}$ in $[\mathrm{P}]$ ): Let $f \in I^{1}(M)$ and denote by $W^{\mathrm{u}}(\Lambda)$ the unstable set of a basic piece $\Lambda$. If $\Lambda_{1}$ and $\Lambda_{2}$ are basic pieces such that $W^{\mathrm{u}}\left(\Lambda_{1}\right) \cap \Lambda_{2} \neq \emptyset$, 
then $\Lambda_{1}$ is expanding. The proof of this result will be omitted because it is similar to that in $[\mathrm{P}]$.

There also exists a partial converse to the previous theorems.

TheOREM C. Let $M$ be a compact manifold and assume that a map $f \in C^{1}(M)$ satisfies the following conditions:

(1) $f$ is Axiom A.

(2) Every critical point of $f$ is wandering.

(3) Every basic piece is expanding or an attracting periodic orbit.

(4) $f^{-1}\left(\Omega^{\prime}(f)\right)=\Omega^{\prime}(f)$, where $\Omega^{\prime}(f)$ denotes the union of the expanding basic pieces of $f$.

(5) $f$ has the no critical relations property.

Then $f$ belongs to $I^{1}(M)$.

This will be proved in the last section. The no critical relations property will be defined later. This condition, and the first two conditions in the statement above, are also necessary for a map to belong to $I^{1}(M)$. The third condition is not necessary: it may happen that an $I^{1}(M)$ map has a saddle type basic piece whose stable and unstable sets do not intersect the critical set. There are no examples known (apart from diffeomorphisms) of $I^{1}(M)$ maps having saddle type basic pieces.

2. Critical sets. The objective of this section is to prove that if $f \in$ $I^{1}(M)$, then every critical point is wandering. The use of the $C^{1}$ topology in the assumption $f \in I^{1}(M)$ is essential. The question whether $f \in I^{r}(M)$ for $r>1$ implies the same conclusion is still open. An affirmative answer would also imply a conjecture stated in [MP]: If $f$ is a $C^{r}$ structurally stable map, then every critical point of $f$ is wandering.

We begin with some definitions and known results.

Definition 2. Let $f$ belong to $C^{r}(M)$.

(1) $S_{k}(f)$ is the set of points $z \in S_{f}$ such that the dimension of the kernel of $D f_{z}$ is equal to $k$.

(2) If $r \geq 2$, a point $z \in S_{k}(f)$ is called generic for $f$ if there exist local charts $\left(U, \tau_{1}\right)$ at $z$ and $\left(V, \tau_{2}\right)$ at $f(z)$ such that the map $x \in$ $\mathbb{R}^{m} \mapsto D\left(\tau_{2}^{-1} f \tau_{1}\right)_{x} \in L\left(\mathbb{R}^{m}\right)$ is transverse to $L_{k}$ (where $L\left(\mathbb{R}^{m}\right)$ is the set of linear maps from $\mathbb{R}^{m}$ to $\mathbb{R}^{m}$, and $L_{k}$ denotes the possibly nonclosed submanifold of $L\left(\mathbb{R}^{m}\right)$ of transformations having kernel of dimension $k$ ).

(3) If $x \in S_{k}(f)$ is generic, then $S_{k}$ is (locally at $x$ ) a codimension $k^{2}$ submanifold of $M$ (see next theorem). A generic critical point $x \in S_{1}(f)$ is of fold type if the kernel of $D f_{x}$ is transverse to $S_{1}(f)$ at $x$. 
Consider the simpler case of a one-dimensional map $f$ : if $x$ is a critical point, then $x$ is generic if and only if the second derivative of $f$ does not vanish at $x$. Now, let $f \in C^{2}(M)$, with $M$ an $m$-dimensional manifold. Suppose that $x \in S_{1}(f)$. Choose coordinates in a neighborhood of $x$ such that the first $m-1$ rows of the differential $D f_{x}$ form a linearly independent set and the last one is $0 \in \mathbb{R}^{m}$. If $f=\left(f_{1}, \ldots, f_{m}\right)$ and $H_{m}$ is the Hessian matrix of $f_{m}$ (the matrix of second derivatives), then $x$ is a generic critical point of $f$ if and only if the $(2 m-1) \times m$ matrix obtained by adding to $H_{m}$ the first $m-1$ rows of $D f_{0}$ has rank $m$.

It was proved by Whitney that around a critical point of fold type a map $f \in C^{\infty}(M)$ is locally geometrically equivalent to the map

$$
q\left(x_{1}, x_{2}, \ldots, x_{m}\right)=\left(x_{1}^{2}, x_{2}, \ldots, x_{m}\right),
$$

acting in $\mathbb{R}^{m}$. The proofs of the statements of the next theorem from differential topology can be found in [GG].

THEOREM 1. Given any manifold $M$, there exists an open and dense set $\mathcal{R}(M) \subset C^{\infty}(M)$ such that, for every $f \in \mathcal{R}(M)$ the following conditions hold:

(1) Each critical point of $f$ is generic.

(2) $S_{1}(f)$ is a codimension one submanifold of $M$, and its closure equals $S_{f}$.

(3) The set of fold type points of $f$ is open and dense in $S_{1}(f)$.

It is not true that the genericity of each critical point of a map $f$ implies that the map belongs to $\mathcal{R}(M)$. However, if $x$ is a fold point of $f \in C^{\infty}(M)$, then there exists a neighborhood $U$ of $x$ such that $f \in \mathcal{R}(U)$ and every critical point of $f$ in $U$ is of fold type. The following semicontinuity holds in general: given a neighborhood $V$ of $S_{f} \cap U$, we have $S_{g} \cap U \subset V$ for every map $g$ in a $C^{1}$ neighborhood of $f$.

The first idea for the proof of Theorem A is quite obvious: the set of critical points can be locally modified in an arbitrary way in $C^{1}$ topology. If one wants to preserve the $C^{\infty}$ genericity of the maps considered, the following can be said.

Lemma 1. Let $x \in S_{f}$ for some $f \in C^{1}(M)$. Given a $C^{1}$ neighborhood $\mathcal{U}$ of $f$ and a codimension one submanifold $N \subset M$ containing $x$, there exists a map $g \in \mathcal{U} \cap \mathcal{R}(M)$ such that $S_{1}(g)$ contains a neighborhood of $x$ in $N$. In addition, the map $g$ can be $C^{\infty}$ approximated by a map $h \in \mathcal{R}(M)$ that is geometrically equivalent to $g$ and such that $S_{h} \cap N$ has empty interior in the relative topology of $N$.

This statement contains the perturbation mechanism that will be needed to obtain a contradiction from the assumption: $f \in I^{1}(M)$ and $S_{f} \cap f^{n}\left(S_{f}\right)$ $\neq \emptyset$. The submanifold $N$ will be $f^{n}\left(S_{f}\right)(n>1)$. 
Some definitions and remarks concerning the structure of critical sets of perturbed maps are in order before proceeding with the proof of this lemma.

REMARK 1. If $x$ is a critical point of fold type of a map $f \in \mathcal{R}$, then the local geometrical equivalence with $q$ implies that there exist neighborhoods $U$ of $x$ and $V$ of $f(x)$ such that $f\left(S_{f}\right)$ separates $V$ into two components $V^{-}$and $V^{+} ; f$ is two-to-one from $U \backslash S_{f}$ onto $V^{+}$and no point in $V^{-}$ has preimages in $U$. If two maps $f$ and $g$ in $\mathcal{R}$ are topologically conjugate $(h f=g h)$ then the homeomorphism $h$ must carry critical points of $f$ to critical points of $g$, because the local forms imply that this is true for fold type points, and the fact that fold type points are dense in $S_{f}$ implies the assertion for the other critical points. Analogously, critical values of $g$ are carried by the conjugacy to critical values of $f$.

This proves an assertion of the introduction: if two topologically equivalent maps belong to $\mathcal{R}$, then they are geometrically equivalent.

The basic idea to prove that no critical point is periodic is the following. It is well known that the maps without critical periodic points constitute a residual set. However, this is not enough for our purposes: we have to find two geometrically equivalent maps close to $f$ such that one of them still has a periodic critical point and the other does not. This will give a contradiction.

It seems intuitively clear that a nongeneric map $f \in C^{1}(M)$ having a nongeneric critical point $x$ can be $C^{1}$ perturbed to a generic map $g \in \mathcal{R}$ for which the same point is still critical. To prove this we make a sequence of perturbations within a given $C^{1}$ neighborhood $\mathcal{U}$ of $f$.

Proposition 1. Let $f$ be a $C^{1}$ map, $\mathcal{U}$ a $C^{1}$ neighborhood of $f$, and $x \in S_{f}$. Then there exists $g \in \mathcal{R}(M) \cap \mathcal{U}$ such that $x$ is a fold type point of $g$. Moreover, if $f$ satisfies one of the following conditions:

(1) $f^{n}(x) \in S_{f}$ for some positive $n$,

(2) $f^{k}(x)$ is periodic for some $k \geq 0$,

(3) $x$ belongs to the stable manifold of a periodic point $p$ of $f$, then the map $g \in \mathcal{R}(M)$ can also be chosen to satisfy the same condition.

Proof. The idea is the following: we first produce a perturbation $\ell$ of $f$ that has a generic critical point at $x$, and then we approximate $\ell$ by a generic map; in this manner, the last perturbation still has a critical point close to $x$ (note that if we begin with a generic perturbation of $f$ using Theorem 1 , then critical points may disappear from a neighborhood of $x$ ).

By using local coordinates one can assume that $M$ is euclidean space and that the critical point $x$ is the origin. Take a one-dimensional subspace $V$ contained in the kernel of $D f_{0}$ and let $H$ be a complementary hyperplane. Now let $A$ be a linear map such that $A(V)=0$ and $A$ is injective in $H$, 
then choose a symmetric linear $B$ such that the critical point 0 of $Q(X)=$ $A X+\langle B X, X\rangle$ is generic (of course an open and dense set of such $B$ exist), and finally use a bump function to define $\ell$ to be equal to $Q$ close to the origin and equal to $f$ outside another neighborhood of the origin. It is clear that $\ell$ is $C^{1}$ close to $f$ and that its critical point 0 is generic.

The final step consists in producing the map $g$. To do this, first perturb $\ell$ to a map $g_{0}$ in $\mathcal{R}$ that is $C^{\infty}$ close to $\ell$ in a neighborhood $U$ of $x$. Because the critical point $x$ was generic for $\ell$, there exists a generic critical point $y \in S_{1}\left(g_{0}\right) \cap U$. Let $\tau$ be a $C^{\infty}$ map close to the identity and carrying $x$ to $y$, and define $g=g_{0} \circ \tau$. This map has the property required in the first statement. The proof that the other properties are preserved by suitable small perturbations is quite similar and relies on the fact that $g(x)=f(x)$ holds by construction, and the perturbations are local, in a small neighborhood of $x$.

The first application of this result is the following:

Proof of Lemma 1. First perturb $f$ to a map (still called $f$ ) in $\mathcal{R}(M) \subset$ $C^{\infty}(M)$ for which $x$ is a critical point, as in the last proposition. As the assertion is local, one can assume without loss of generality that $M=\mathbb{R}^{m}$ and that 0 is a critical point of $f$. It can also be assumed that $N=\{x=$ $\left.\left(x_{1}, \ldots, x_{m}\right) \in \mathbb{R}^{m} \mid x_{m}=0\right\}$, because there exists a diffeomorphism $T$ carrying $N$ to this set and the origin to itself. In addition, if $L$ is an appropriate linear isomorphism, then $L \circ D f_{0} \circ D T_{0}^{-1}$ is a linear map whose matrix in canonical coordinates is diagonal, has the first $m-1$ rows linearly independent and the last one null. Maintain the notation $f$ for the map in these coordinates, so that it satisfies all the conditions.

These assumptions imply that the last coordinate of $f$ satisfies $f_{m}(x)=$ $f_{m}(0)+r(x)$ for some $C^{1}$ function $r$ such that $|r(x)| /\|x\| \rightarrow 0$ as $x \rightarrow 0$. Given any $\varepsilon>0$ there exist a number $\varrho \in(0, \varepsilon / 2)$ and a function $\varphi=\varphi_{\varepsilon}$ : $\mathbb{R} \rightarrow[0,1]$, of class $C^{\infty}$, that is equal to 0 in $|x| \leq \varrho$, equal to 1 outside $|x|<\varepsilon$, and such that $\left|\varphi^{\prime}(x)\right|<2 / \varepsilon$. Let

$$
g_{m}\left(x_{1}, \ldots, x_{m}\right)=f_{m}(0)+\varphi(\|x\|) r(x)+(1-\varphi(\|x\|)) \varepsilon x_{m}^{2}
$$

and define $g=\left(f_{1}, \ldots, f_{m-1}, g_{m}\right)$. Note that

$\nabla g_{m}(x)=\varphi^{\prime}(\|x\|) \frac{r(x)-\varepsilon x_{m}^{2}}{\|x\|} x+\varphi(\|x\|) \nabla r(x)+2(1-\varphi(\|x\|)) \varepsilon\left(0, \ldots, 0, x_{m}\right)$.

Note that for $\|x\| \leq \varrho$, the determinant of $D g_{x}$ is equal to 0 if and only if $x_{m}=0$ (because the assumptions on $f$ imply that the upper left $(m-1) \times$ $(m-1)$ block in the matrix of $D f_{x}$ is nonsingular). Hence $S_{g} \cap B(0 ; \varrho) \subset N$, where $B(0 ; \varrho)$ is the ball centered at the origin and with radius $\varrho$. It remains to prove that $\varepsilon$ can be chosen so that $g \in \mathcal{U}$. Note that $f(x)=g(x)$ for 
$x \notin B(0 ; \varepsilon)$ and that in this ball we have

$$
\left|g_{m}(x)-f_{m}(x)\right| \leq|\varphi(\|x\|)-1|\left(\|r(x)\|+\varepsilon\|x\|^{2}\right) .
$$

Hence $g$ is $C^{0}$ close to $f$ if $\varepsilon$ is small. Finally, note that

$\left\|\nabla g_{m}(x)-\nabla f_{m}(x)\right\| \leq(2 / \varepsilon)\left|r(x)-\varepsilon x_{m}^{2}\right|+|\varphi(\|x\|)-1|(\|\nabla r(x)\|+2 \varepsilon\|x\|)$.

Observe that given any $\delta>0$, one can choose $\varepsilon$ such that $|r(x)| \leq \delta\|x\|$ and $\|\nabla r(x)\| \leq \delta$ for every $x \in B(0 ; \varepsilon)$. It follows that the $C^{1}$ distance between $f$ and $g$ is at most $2\left(\delta+\varepsilon^{2}\right)+\delta+2 \varepsilon^{2}$. It remains to show that $g \in \mathcal{R}$. Observe that by construction, the critical point at the origin is generic: indeed, the Hessian matrix of $g_{m}$ at the origin has entries $a_{i j}$, where $a_{i j}=0$ for every $(i, j) \neq(m, m)$, and $a_{m m}=2$, but the set of vectors $\left\{\nabla f_{1}(0), \ldots, \nabla f_{m-1}(0),(0, \ldots, 0,2)\right\}$ is linearly independent, by the choice of local coordinates. This implies that $g \in \mathcal{R}(U)$ for some neighborhood $U$ of the origin. On the other hand, as $f$ was taken in $\mathcal{R}(M)$, it follows that $g \in \mathcal{R}\left(B^{c}\right)$, where $B^{c}$ is the complement of $B(0 ; \varepsilon)(f$ and $g$ coincide there). Take a bump function $\theta$ equal to 0 in the complement of $U \cap B(0 ; \varrho)$ and to 1 in a neighborhood $V$ of $x$. Let $d$ be the $C^{\infty}$ distance fom $\theta$ to the null function. As $\mathcal{R}(M)$ is dense in $C^{1}(M)$, one can choose any $g_{1} \in \mathcal{R}(M)$ such that the $C^{\infty}$ distance between $g$ and $g_{1}$ is less than $\tau / d$ (where $\tau$ is taken such that every map in the ball of center $g$ and radius $\tau$ belongs to $\mathcal{R}(U)$ ). Finally, define $g_{0}=\theta g+$ $(1-\theta) g_{1}$. This $g_{0}$ satisfies all conditions: it belongs to $\mathcal{R}(U)$, because it is $\tau$-close to $g$ there; it belongs to $\mathcal{R}\left(U^{c}\right)$ because it is equal to $g_{1}$ there; it satisfies $S_{g_{1}} \cap V=N \cap V$, because in that neighborhood it coincides with $g$.

It remains to prove the second assertion of the lemma. Let $\varphi$ be a $C^{\infty}$ diffeomorphism of $M$ such that $\varphi(x)=x, \varphi(V)=V$ and $\varphi^{-1}(N) \cap N$ does not contain a neighborhood of $x$ in $N$. It is clear that such a map can be obtained arbitrarily $C^{\infty}$ close to the identity (for example, $D \varphi_{0}$ can take $T_{0} N$ to any other hyperplane contained in $\left.T_{0} M\right)$. Observe that $h=g \varphi$ is $C^{\infty}$ close to $g$, so that it belongs to $\mathcal{U} \cap \mathcal{R}(M) ; h$ is geometrically equivalent to $g$ (because $i h=g \varphi$, where $i$ is the identity map) and $S_{h} \cap V=\varphi^{-1}\left(S_{g} \cap V\right)$, so that $S_{h}$ cannot contain a neighborhood of $x$ in $N$.

REMARK 2. A point $x$ is preperiodic for $f$ if there exist $k \geq 0$ and $p>0$ such that $f^{k+p}(x)=f^{k}(x)$. If $k=0$ then $x$ is periodic. The period of a preperiodic point is the minimum $p$ satisfying the above equation. Denote by $P_{k, p}(f)$ the set of points $x$ such that the above holds and $k$ is minimum, and by $\operatorname{Per}(f)$ the union of the $P_{0, p}(f)$ for positive $p$.

A periodic point $x$ of period $p$ is hyperbolic if the differential of $f^{p}$ at $x$ has no eigenvalue null or of modulus one. It is well known that given any $p \geq 1$, the set $\mathcal{G}_{p}$ of maps for which every periodic point of period at most 
$p$ is hyperbolic, is open and dense in every $C^{r}(M)$. For every map in this open and dense set, the number of periodic points of period at most $p$ is finite and locally constant. It is clear that if $f \in \mathcal{G}_{p}$ then $P_{0, p}(f) \cap S_{f}=\emptyset$. This argument can be completed to obtain an open and dense set $\mathcal{G}_{k, p}$ of maps such that $P_{k, p}(f) \cap S_{f}$ is empty.

On the other hand, assume that a map $f \in C^{1}(M)$ has a critical point $x \in P_{k, p}(f)$. The second assertion of Proposition 1 gives a map $g \in$ $\mathcal{R}(M) \cap \mathcal{G}_{p}, C^{1}$ close to $f$, such that $x$ still belongs to $P_{k, p}(g) \cap S_{g}$.

Lemma 2. If $f \in I^{1}(M)$, then no preperiodic point of $f$ is critical.

Proof. Note that intersections between $P_{k, p}(f)$ and $S_{f}$ can be avoided by small perturbations; what must be proved now is that this can be done within the same class of geometric equivalence. Therefore one can assume that $x \in S_{1}(f)$ is an isolated point of $P_{k, p}(f)$, that $f^{k}(x)$ is hyperbolic, and that $f \in \mathcal{R}(M)$. We will arrive at a contradiction if we can find a map $g$ that is $C^{1}$ close to $f$, geometrically equivalent to $f$ and such that no critical point of $g$ belongs to $P_{k, p}(g)$.

This will be done in local charts. Let $\left(U, \tau_{1}\right)$ and $\left(V, \tau_{2}\right)$ be local charts at $x$ and $f(x)$ respectively. The local coordinates can be chosen so that $\tilde{f}\left(x_{1}, \ldots, x_{m}\right)=\tau_{2} f \tau_{1}^{-1}\left(x_{1}, \ldots, x_{m}\right)=\left(x_{1}^{2}, \ldots, x_{m}\right)$. Let $h\left(x_{1}, \ldots, x_{m}\right)=$ $\left(h_{1}(x), x_{2}, \ldots, x_{m}\right)$, where $h_{1}(x)=x_{1}^{2}-\varepsilon \varrho(\|x\|) x_{1}, \varepsilon$ is an arbitrary positive number and $\varrho$ satisfies the following conditions: The function $\varrho$ is $C^{\infty}$, $\varrho(0)=1, \varrho(x)=0$ for every $|x|>1$ and the $C^{2}$ norm of $\varrho$ is less than a constant $k$. The map $g$ will be $\tau_{2}^{-1} h \tau_{1}$. To see that $g$ satisfies the above condition we note that $h$ is $C^{1}$ close to $\tilde{f}$ if $\varepsilon$ is small enough, that $g(x)=f(x)$ and that $x$ is not a critical point of $g$; hence $P_{k, p}(g) \cap S_{g}=\emptyset$. It remains to show that $f$ and $g$ are geometrically equivalent and for this it is enough to prove that $\tilde{f}$ and $h$ are geometrically equivalent.

The equation of the critical points of $h$ is $\partial_{1} h_{1}(x)=0$; as $\partial_{11} h_{1}$ is close to 2 if $\varepsilon$ is small, it follows by the implicit function theorem that there exists a $C^{\infty}$ function $c\left(x_{2}, \ldots, x_{m}\right)$ whose graph is the set of critical points of $h$. Define

$$
\psi(y)=\left(y_{1}-h_{1}\left(c\left(y_{2}, \ldots, y_{m}\right), y_{2}, \ldots, y_{m}\right), y_{2}, \ldots, y_{m}\right)
$$

so that

$$
\psi\left(h_{1}(x), x_{2}, \ldots, x_{m}\right)=\left(h_{1}(x)-h_{1}\left(c\left(x_{2}, \ldots, x_{m}\right), x_{2}, \ldots, x_{m}\right), x_{2}, \ldots, x_{m}\right) .
$$

If $\varphi_{1}(x)$ is a function such that $\varphi_{1}^{2}(x)=h_{1}(x)-h_{1}\left(c\left(x_{2}, \ldots, x_{m}\right), x_{2}, \ldots, x_{m}\right)$ then $\tilde{f} \varphi=\psi h$, where $\varphi(x)=\left(\varphi_{1}(x), x_{2}, \ldots, x_{m}\right)$. It remains to prove that $\varphi$ is a $C^{\infty}$ diffeomorphism. Indeed,

$h_{1}(x)-h_{1}\left(c\left(x_{2}, \ldots, x_{m}\right), x_{2}, \ldots, x_{m}\right)=\alpha\left(x_{1}, \ldots, x_{m}\right)\left(x_{1}-c\left(x_{2}, \ldots, x_{m}\right)\right)^{2}$

with $\alpha$ a positive $C^{\infty}$ function. 
Proposition 2. If $f \in I^{1}(M)$, then there exist neighborhoods $\mathcal{U}$ of $f$ and $U$ of $S_{f}$ such that $U \cap h^{n}(U)=\emptyset$ for every $n \geq 1$ and every $h \in \mathcal{U}$.

Proof. We first claim that $f^{n}\left(S_{f}\right)$ does not intersect $S_{f}$ if $n \geq 1$.

Assume by contradiction that $f \in I^{1}(M)$, and that there exists a point $x \in S_{f}$ such that $f^{n}(x) \in S_{f}$ for some (minimum) $n>0$. By Proposition 1, one can assume that $f \in \mathcal{R}$ and $x \in S_{1}(f)$. Let $\left\{U_{j} \mid 0 \leq j \leq n\right\}$ be a disjoint sequence of open sets such that each $U_{j}$ is a neighborhood of $f^{j}(x)$ and $f\left(U_{j}\right) \subset U_{j+1}$. This is possible since $x$ is not preperiodic.

Note then that $f^{n}\left(S_{f}\right)$ is a codimension one submanifold of $M$ containing $f^{n}(x)$; indeed, $f\left(S_{f} \cap U_{0}\right)$ is a submanifold since the restriction of $f$ to $S_{1}(f)$ is an immersion whenever $x$ is a fold point. Then the fact that $D f_{f^{j}(x)}$ is an immersion for every $j \geq 1$ implies the assertion.

Now apply Lemma 1. The first assertion there gives a map $g \in \mathcal{R}$ for which $S_{g}$ contains a neighborhood of $g^{n}(x)$ in $N=f^{n}\left(S_{f} \cap U_{0}\right)$ (note that the support of this perturbation is contained in $U_{n}$, so $f^{n}\left(S_{f} \cap U_{0}\right) \cap U_{n}=$ $\left.g^{n}\left(S_{g} \cap U_{0}\right) \cap U_{n}\right)$. The second perturbation in Lemma 1 gives a map $h$ for which $S_{h} \cap h^{n}\left(S_{h}\right)$ has empty interior in the submanifold $h^{n}\left(S_{h} \cap U_{n}\right)$. The support of this last perturbation is also contained in $U_{n}$, hence the set of critical points in $U_{0}$ and their images until $n$ are the same for $f$, $g$ and $h$. A contradiction follows, because, on one hand, $g$ and $h$ must be topologically conjugate since Lemma 1 says that $g$ and $h$ are geometrically equivalent $C^{1}$ perturbations of $f$, and on other hand, $g$ and $h$ cannot be topologically conjugate since such a conjugacy must carry points in the interior of $S_{g} \cap g^{n}\left(S_{g}\right)$ (a nonempty set) to points in the interior of $S_{h} \cap h^{n}\left(S_{h}\right)$ (empty). This proves the claim.

To prove the assertion of the proposition, we use the following version of Franks' lemma $([\mathrm{F}])$, whose proof is exactly the same:

Let $f$ be a $C^{1}(M)$ map and $\mathcal{U}_{0}$ a $C^{1}$ neighborhood of $f$. Then there exist a $C^{1}$ neighborhood $\mathcal{U}_{1}$ of $f$ and a positive number $\delta$ with the following property. If $x \in M$ and $n$ is a positive integer, and $T_{i}$ is a sequence of linear maps such that for $i \leq n$ we have $\left\|T_{i}-D g_{x_{i}}\right\|<\delta$ for some $g \in \mathcal{U}_{1}$, where $x_{i}=g^{i}(x)$ and $x_{i} \neq x_{j}$ whenever $i \neq j$, then there exists $g_{1} \in \mathcal{U}_{0}$ such that $D\left(g_{1}\right)_{x_{i}}=T_{i}$ and $g_{1}^{i}(x)=g^{i}(x)$ for every $i \leq n$.

Let $\mathcal{U}_{0}$ be a neighborhood of $f$ such that every map in $\mathcal{U}_{0}$ belongs to $I^{1}(M)$. Let $\mathcal{U}_{1}$ and $\delta$ be as above, let $U$ be a neighborhood of $S_{f}$ and let $\mathcal{U}_{2} \subset \mathcal{U}_{1}$ be such that for every $x \in U$ and every $g \in \mathcal{U}_{2}$ there exists a unit vector $v \in T_{x} M$ such that $\left\|D g_{x}(v)\right\|<\delta$. If the conclusion of the proposition is false, then there exists a map $g \in \mathcal{U}_{2}$, a point $x_{0}$ and an integer $n$ such that $x_{0}$ and $g^{n}\left(x_{0}\right)$ belong to $U$. Let $v_{0}$ and $v_{n}$ be such that $\left\|D g_{x_{0}}\left(v_{0}\right)\right\|$ and $\left\|D g_{x_{n}}\left(v_{n}\right)\right\|$ are both smaller than $\delta$. For $i \leq n$, let $T_{i}$ be linear transformations such that $T_{0}\left(v_{0}\right)=0, T_{n}\left(v_{n}\right)=0$, any other $T_{i}$ is equal to $D g_{x_{i}}$, and 
$\left\|T_{i}-D g_{x_{i}}\right\|<\delta$. Now the map $g_{1}$ given by Franks' lemma contradicts the first claim of the proof.

We have the desired conclusion:

Corollary 1. If $f \in I^{1}(M)$, then $\Omega(f) \cap S_{f}=\emptyset$.

We finish this section with another perturbation result.

LEMMA 3. Let $M$ be a compact manifold, $f$ be a map in $I^{1}(M)$ and $W$ a neighborhood of $S_{f}$. There exists a $C^{1}$ neighborhood $\mathcal{U}$ of $f$ such that if a map $g \in \mathcal{U}$ is equal to $f$ in $W$, then $f$ and $g$ are geometrically equivalent.

Proof. We first claim that there exist $\varrho>0$ and a $C^{1}$ neighborhood $\mathcal{U}_{0}$ of $f$ such that for every $x \in W^{c}$ (the complement of $W$ ) and $g \in \mathcal{U}_{0}$ the restriction of $g$ to the ball $B(x ; \varrho)$ is one-to-one. This is clear, since for every $g$ in a $C^{1}$ neighborhood of $f$, the norm of the inverse of $D g_{x}$ at $x \in W^{c}$ is uniformly bounded.

The claim implies that the distance between two different preimages of the same point is at least $\varrho$, so both preimages belong to $W$. To prove the lemma, $C^{1}$ diffeomorphisms $\varphi$ and $\psi$ must be found such that $f \varphi=\psi g$. Take $\psi$ equal to the identity map. Then define $\varphi$ to be the identity in $W$ and $f^{-1} g$ in $W^{c}$. To ensure that $\varphi$ is a diffeomorphism close to the identity, one must choose an appropriate branch of $f^{-1}$, which is possible by the claim.

\section{Hyperbolicity}

Lemma 4. If $f \in I^{1}(M)$ then every periodic point of $f$ is hyperbolic.

Proof. Suppose that $f$ has a nonhyperbolic periodic point $x$ with period $n$. Let $g$ be a map in $\mathcal{R}$ such that $x$ is periodic nonhyperbolic for $g$, has period $n$ and every other periodic point of $g$ of period less than or equal to $n$ is hyperbolic. To find $g$, first perturb $f$ to a map such that the periodic point $x$ is nonhyperbolic but is isolated in the set of periodic points of period $n$ of $f$. Then apply the usual mechanisms to make the other periodic points of period at most $n$ hyperbolic. Now we construct two $C^{\infty}$ maps, arbitrarily $C^{1}$ close to $f$, such that the periodic point $x$ is hyperbolic for both maps but has different character (the dimension of the stable space changes) and the perturbation has support outside the set of critical points of $f$. By Lemma 3 these maps are geometrically equivalent, which contradicts the fact that $f \in I^{1}(M)$.

Proof of Theorem A. By Corollary 1 no critical point is wandering. By Lemma 4, every periodic point is hyperbolic. Now we use Theorem A of Aoki, Moriyazu and Sumi [AMS], which implies the following: 
If a map $f$ with $S_{f} \cap \Omega(f)=\emptyset$ has a $C^{1}$ neighborhood contained in the set of mappings having every periodic point hyperbolic, then the nonwandering set of $f$ has a hyperbolic structure and the set of periodic points of $f$ is dense in the nonwandering set of $f$.

As shown by Przytycki [P], this is not enough to obtain the $C^{1} \Omega$-stability of $f$ : for this it will be necessary to show first that each basic piece is either expanding or injective. We will prove the $\Omega$-stability of $f$ directly from the definition.

Let $\mathcal{U}$ be the neighborhood of $f$ given by the definition of $I^{1}(M)$ and $U$ such that the conclusion of Proposition 2 holds for $\mathcal{U}$ and $U$. Let $W$ be a neighborhood of $S_{f}$ whose closure is contained in $U$. There exists a $C^{1}$ neighborhood $\mathcal{U}_{0} \subset \mathcal{U}$ of $f$ such that:

(1) The conclusions of Lemma 3 hold for the neighborhoods $W$ of $S_{f}$ and $\mathcal{U}_{0}$ of $f$.

(2) If $f_{1}$ and $f_{2}$ belong to $\mathcal{U}_{0}$ then there exists a map $F=F\left(f_{1}, f_{2}\right) \in \mathcal{U}$ such that $F$ is equal to $f_{1}$ in $W$ and equal to $f_{2}$ in the complement of $U$.

Let $\mathcal{U}_{1} \subset \mathcal{U}_{0}$ be a neighborhood of $f$ such that $F\left(f_{1}, f_{2}\right) \in \mathcal{U}_{0}$ whenever $f_{1}$ and $f_{2}$ belong to $\mathcal{U}_{1}$.

Let $g \in \mathcal{U}_{1}$, and $h=F(g, f) \in \mathcal{U}_{0}$. By Lemma 3, $g$ and $h$ are topologically equivalent. By Proposition 2, the periodic points of $f$ and $h$ are contained in $U^{c}$, where the maps coincide. It follows that $\operatorname{Per}(f)=\operatorname{Per}(h)$, which implies that $f=h$ in $\Omega(f)=\Omega(h)$, and we conclude that $f$ and $h$ are $\Omega$-equivalent.

4. Location of critical sets. In this section we prove Theorem B. It was already shown that every critical point of an $f \in I^{1}(M)$ is wandering, and that $f$ is an Axiom A map. It follows that every point is contained in the stable set of some basic piece and in the unstable set of a basic piece. It will be shown that a basic piece whose stable (resp. unstable) set intersects $S_{f}$ must be a periodic attractor (resp. an expanding set). Indeed, if this is not the case, and a stable or unstable manifold of a basic piece of another type contains a critical point, then this critical point can be perturbed in the same class of geometric equivalence in order to produce some nonequivalent dynamical consequences.

We refer the reader to the article of Przytycki $[\mathrm{P}]$ for the definitions of stable and unstable sets and properties of Axiom A maps. It is clear, and will be used below, that $P_{k, p}(f)$ is an invariant of topological conjugacy, as also is the union of the stable (resp. unstable) sets of its points. 
The idea is the following: Let $z$ be a generic critical point of fold type of $f$ and assume that it belongs to the stable manifold of a basic piece that is not an attracting periodic orbit. By the density of periodic points in each basic piece, it can be assumed without loss of generality that there exists a periodic point $x$ of $f$ whose stable manifold contains $z$.

A first lemma will be needed to show that by means of a $C^{1}$ perturbation, one can create a map $g$ having a segment $L$ close to $z$ such that the image of $L$ is a single point. If maps $g_{1}$ and $g_{2}$ are geometrically equivalent to $g$, then there exists a segment $L_{1}$ (resp. $L_{2}$ ) where $g_{1}$ (resp. $g_{2}$ ) is constant. Then $g_{1}$ and $g_{2}$ are topologically equivalent, and the conjugacy must send $L_{1}$ to $L_{2}$ and $g_{1}\left(L_{1}\right)$ to $g_{2}\left(L_{2}\right)$.

A contradiction will be found if one can put the point $g_{1}\left(L_{1}\right)$ in the stable manifold of the $g_{1}$-periodic point $x$ and the point $g_{2}\left(L_{2}\right)$ outside the stable manifold of a periodic point of period equal to that of $x$. This will be possible since, by assumption, the periodic point $x$ of $f$ was not an attractor. The proof for the repelling case uses a similar argument.

Note that by Proposition 1, one can choose the map $f$ in $\mathcal{R}$, preserving its other properties.

Lemma 5. Let $z$ be a fold point of a map $f$ in $\mathcal{R}, \mathcal{U}$ a $C^{1}$ neighborhood of $f$ and $U$ a neighborhood of $z$. Then there exists a map $g \in \mathcal{U}$ and $a$ segment $L \subset U$ such that $S_{g}=S_{f} \cup L$ and $L \cap S_{f}=\emptyset$. Moreover, $g$ is constant in $L$.

A segment is the image of a smooth injective curve $\alpha:[0,1] \rightarrow M$.

Proof. One can assume that $f\left(x_{1}, \ldots, x_{m}\right)=\left(x_{1}^{2}, x_{2}, \ldots, x_{m}\right)$ and that the point $z$ is the origin. Let $\varepsilon$ be a positive number.

A point $X \in \mathbb{R}^{m}$ will be denoted by $X=(x, Y)$, where $x \in \mathbb{R}$ and $Y \in \mathbb{R}^{m-1}$. Given a segment $I \subset \mathbb{R}^{+}$, let $Q_{1}(x)=2 x$ and take a $C^{1}$ function $Q_{0}$ defined in $\mathbb{R}$ such that $Q_{0}(x)=0$ if and only if $x \in I$ or $x=0$. If $I$ was the segment $[a, b]$, one can take $b<\varepsilon / 2$ ( $a$ is positive) to obtain a function $Q_{0}$ that is $\varepsilon-C^{0}$ close to the function $Q_{1}$. Assume also that $Q_{0}(x)=2 x$ for every $x \notin[0,1]$, that $Q_{0}(x) \geq 0$ for every $x>0$, and that $\int_{0}^{1} Q_{0}=1$. Let $\left\{Q_{y}: y \in[0,1]\right\}$ be a homotopy from $Q_{0}$ to $Q_{1}$ such that the following conditions hold:

(1) $Q_{y}$ is $\varepsilon-C^{0}$ close to $Q_{1}$ for every $y$.

(2) $\int_{0}^{1} Q_{y}=1$ for every $y$.

(3) For every $x \notin[0,1], Q_{y}(x)=2 x$.

(4) If $y>0$ then $Q_{y}(x)>0$ for every $x>0$.

(5) For every $x$ fixed the function $y \mapsto Q_{x}(y)$ is $C^{1}$, with derivative smaller than $3 b$. 
For $y>1$ set $Q_{y}=Q_{1}$. Next define, for every $Y \in \mathbb{R}^{m}$, the function

$$
g_{1}(x, Y)=\int_{0}^{x} Q_{|Y|}(t) d t .
$$

We now claim that $g(x, Y)=\left(g_{1}(x, Y), Y\right)$ satisfies the assertions of the lemma if $L$ is defined to be $I \times\{0\}$. Indeed, $\partial_{1} g_{1}(x, Y)=Q_{|Y|}(x)$ is equal to 0 if and only if $x=0$ or $(x, Y) \in L$ (by (4)). Conditions (2) and (3) imply that $g(x, Y)=f(x, Y)$ whenever $x \notin[0,1]$ or $|Y|>1$. It follows from properties (1) and (5) that $g$ is $3 b-C^{1}$ close to $f$.

Note that $g(L) \notin g\left(S_{g} \backslash L\right)$ because $Q_{0}$ is positive in $(0, a)$.

Proof of Theorem B. Part 1. The map $f$ belongs to $I^{1}(M)$, and has a critical point $z$ contained in the stable manifold $W^{\mathrm{s}}(x, f)$ of a fixed point $x$ that is not an attractor. It can also be assumed that $f$ is generic and that $z$ is a fold type point.

To produce a perturbation $g_{1}$ of $f$ such that $L \cap W^{\mathrm{s}}\left(x, g_{1}\right) \neq \emptyset$, just take the map $g$ of the previous lemma. If the neighborhood $U$ was taken such that its forward iterates under $f$ do not intersect $U$, then $g(L)=f(z)$ belongs to the stable manifold of $x$.

The construction of the map $g_{2}$ is not so easy, because the intersection of the stable manifold of $x$ with the neighborhood $U$ can have infinitely many components. Consider first the case where the basic piece that contains the periodic point $x$ is not an attractor. The same proof made for Axiom A diffeomorphisms can be adapted to show that the union of the basins of the attracting basic pieces is open and dense. Before applying the lemma, perturb the map $f$ in $U$ so that $z$ belongs to the basin of an attractor, without changing the class of geometric equivalence $f$ nor the condition that $z$ is a critical point. Indeed, let $\tau$ be a translation supported in a small neighborhood $W$ of $f(U)$ (that is not necessarily open) such that $\tau(f(z)) \in B$ where $B$ is equal to the intersection of the basin of an attractor with $f(U)$. Define the new map $f^{\prime}$ as follows: if $y \in U$, then $f^{\prime}(y)=\tau(f(y))$, and if $y \notin U$, then $f^{\prime}(y)=f(y)$. Note that if $W$ is sufficiently small, then the preimages of $W$ are disjoint open sets, so $f^{\prime}$ is well defined, smooth, and close to $f$. Moreover, by the choice of $U$ (disjoint from its forward iterates) the set $B$ is still contained in the basin of an attractor of $f^{\prime}$. Now one can apply the lemma, with $L$ contained in the basin ot the attractor in question, giving a map $g_{2}$ that is geometrically but not topologically equivalent to $g_{1}$.

To treat the remaining case, assume $\Lambda$ is an attracting basic piece and $x \in \Lambda$. Let $W$ be a neighborhood of $\Lambda$ such that $f(W) \subset W$ and $f$ is injective in $W$ ( $f$ is injective in $\Lambda$ because $f$ is $\Omega$-stable). Let $g$ be a map as in the lemma so that $L$ is contained in the basin of $\Lambda$. Let $k>0$ be the first positive integer such that $y=g^{k}(L) \in W$; as periodic points are dense in $\Lambda$, 
one can perturb $g$ in a neighborhood of $\Lambda$ to a map $g_{2}$ such that $y$ belongs to the stable manifold of a periodic point of period greater than that of $x$. This last perturbation must be geometrically equivalent to $g_{1}$, but cannot be topologically equivalent. This proves the first assertion in part 1 . Let $C$ be a component of $S_{f}$ that intersects the basin $B$ of an attracting periodic orbit. As $B$ is open and its boundary does not intersect the basin of another attractor, it follows that $C \cap \partial B=\emptyset$, so $C \subset B$.

Part 2. The map $f$ belongs to $I^{1}(M)$ and there exists a nonrepelling fixed point $x$ whose unstable set contains a critical point $z$. The unstable set is defined as the union of the images of a local unstable manifold. Now there exists a neighborhood $U$ of $z$ such that the future images of $U$ do not intersect $U$. This implies that a perturbation of $f$ in $U$ does not produce any change in $W^{\mathrm{u}}(x, f) \cap U$. So one can find a perturbation such that both extreme points of $L$ are contained in the complement of $W^{\mathrm{u}}$ and another perturbation such that at least one extreme point of $L$ belongs to $W^{\mathrm{u}}$. Finally, to prove the second assertion in this part, assume that $C$ intersects the unstable set $W$ of an expanding piece $\Lambda$. Now $W$ is not necessarily open, but its boundary is contained in the set $\bigcup_{n>0} f^{n}\left(S_{f}\right)$. By Proposition 2 it follows that $C \cap \partial W=\emptyset$, so $C \subset W$.

5. Sufficient conditions and examples. This section contains the proof of the partial converse, Theorem $\mathrm{C}$ of the introduction. In [IPR1], the existence of $C^{3}$ structurally maps was shown. These were perturbations of complex polynomials, so the components of the set of critical points were arbitrarily small, which makes the proof simpler than the one presented here, which follows the same ideas.

Definition 3. A $C^{1}$ map $f$ has the no critical relations property if there exist open connected sets $U_{1}, \ldots, U_{n}$ such that:

(1) $S_{f} \subset \bigcup_{i} U_{i}$.

(2) The closures of the sets $U_{i}$ are disjoint.

(3) Given nonnegative integers $j$ and $l$ such that $f^{j}\left(U_{k}\right) \cap f^{l}\left(U_{i}\right) \neq \emptyset$, then $j=l$ and $k=i$.

(4) The restriction of $f$ to the closure of $f^{j}\left(U_{i}\right)$ is injective for every $j>0$ and $1 \leq i \leq n$.

It is important to note that items (3) and (4) do not represent an infinite number of conditions, since, under the hypothesis of Theorem C, each component of $S_{f}$ is entirely contained in the basin of a unique attracting periodic orbit and hence is eventually contained in an open set where the map is injective. Therefore, each $U_{i}$ is contained in a component of the basin of an attractor. 
Denote by $B_{f}$ the union of the basins of the periodic attractors of $f$. Define also the Julia set of $f$ to be the set of nonwandering points of $f$ that are not periodic attractors. This set was denoted by $\Omega^{\prime}(f)$ before. The first assertion describes global aspects of the dynamics of every $g$ in a neighborhood of $f$.

Lemma 6. If $f$ satisfies the hypothesis of Theorem $C$, then:

(1) $M=B_{f} \cup \Omega^{\prime}(f)$.

(2) Either $B_{f}$ is empty (and $f$ is an expanding map) or $M=\bar{B}_{f}$ (the bar denoting closure).

(3) $f$ is $C^{1} \Omega$-stable, and hence the same conclusions hold for every map $g$ in a $C^{1}$ neighborhood of $f$.

Proof. Assume that there exists a point $x \in M \backslash B_{f}$ and let $U$ be a neighborhood of $x$. As the sequence $\left\{f^{n}(x)\right\}$ converges to $\Omega(f)$, there exists an $m>0$ such that $f^{m}(x) \in \Omega^{\prime}(f)$ and hence $x \in \Omega^{\prime}(f)$ by assumption (4). This proves the first item. If $U \subset \Omega^{\prime}(f)$ then $\Omega^{\prime}(f)=M$, hence $B_{f}$ is empty or $U \cap B_{f} \neq \emptyset$.

As $f$ is Axiom A, has no cycles by hypothesis (3) of Theorem C, and every critical point is wandering, the theorem of Przytycki implies that $f$ is $C^{1} \Omega$-stable.

There exists a uniform expansiveness constant for the restrictions of the maps $g$ in a neighborhood of $f$ to the respective Julia sets. Let $\varepsilon$ be this constant. Let $\alpha>0$ be less than $\varepsilon$ and less than the distance between different $U_{i}$ 's. Let $\mathcal{U}$ be a $C^{1}$ neighborhood of $f$ such that every $g \in \mathcal{U}$ is $\Omega$-equivalent to $f$ and $S_{g} \subset \cup U_{i}$. The number $\alpha$ and neighborhood $\mathcal{U}$ will be diminished later. Take $g_{1}$ and $g_{2}$ in $\mathcal{U}$ that are $\mathcal{Z}$-geometrically equivalent, where $\mathcal{Z}$ is the $C^{0}$ neighborhood of the identity of size $\alpha$. One has

$$
\varphi g_{1}=g_{2} \psi
$$

where the distance from $\psi(x)$ and $\varphi(x)$ to $x$ is less than $\alpha$ for every $x \in M$.

Proof of Theorem $C$. The idea is to construct a conjugacy $h$ from $B_{g_{1}}$ to $B_{g_{2}}$ that is $\varepsilon-C^{0}$ close to the identity in a neighborhood of $\Omega^{\prime}\left(g_{1}\right)$, and then continuously extend it to the closure of $B_{g_{1}}=M$, using the fact that $\Omega^{\prime}\left(g_{1}\right)$ is expanding.

Construction of a fundamental domain. Let $x$ be an attracting periodic point of $f$; assume that $x$ is fixed to simplify notation. Let $V$ be a neighborhood of $x$ such that the closure of $f(V)$ is contained in $V$ and $f$ restricted to $V$ is injective. The first step is to construct an open set $V^{\prime} \subset V$ with the same properties of $V$ and such that for every $U_{i}$ contained in the basin of $x$ there exists a positive integer $n_{i}$ such that $f^{n_{i}}\left(U_{i}\right)$ is contained in the 
interior of the fundamental domain $V^{\prime} \backslash f\left(V^{\prime}\right)$. Assume first that only one of the sets $U_{i}$ is contained in the basin of $x$. Let $n$ be the minimum positive integer such that the closure $W$ of $f^{n}\left(U_{i}\right)$ is contained in $V$. As $x$ cannot belong to $W$, there exist a finite number of forward iterates of $V$ that intersect $W$, say $f^{p}(V) \cap W$ is empty for every $p>N$. Define a sequence $\left\{W_{0}, W_{1}, \ldots, W_{N}\right\}$ of compact sets such that:

- $W_{0}=W$.

- $W_{k}$ is contained in the interior of $W_{k+1}$ for every $k=0, \ldots, N-1$.

- $W_{N}$ is contained in $V$ and does not intersect $f^{p}(V)$ for any $p>N$.

- $f\left(W_{N}\right) \cap W_{N}=\emptyset$.

Then we define

$$
V^{\prime}=V \backslash \bigcup_{k=1}^{N} f^{-k}\left(W_{k}\right),
$$

and prove that $V^{\prime}$ satisfies the above claim. Indeed, if $x \in V$, then $f(x)$ belongs to the interior of $V$; if, in addition, $x \notin f^{-k}\left(W_{k}\right)$, then $f(x)$ does not belong to $f^{1-k}\left(W_{k}\right)$, whose interior contains $f^{1-k}\left(W_{k-1}\right)$, because $f$ is a diffeomorphism in $V$. This proves that $V^{\prime} \backslash f\left(V^{\prime}\right)$ is a fundamental domain for $f$. Finally, if $y \in V^{\prime}$, then $y \notin f^{-1}\left(W_{1}\right)$, which contains $f^{-1}(W)$ in its interior; this implies that $W$ is contained in the interior of the fundamental domain.

Assume now that $U_{1}, \ldots, U_{L}$ are contained in the basin of $x$, and let $n_{i}$ be such that $f^{n_{i}}\left(U_{i}\right)$ is contained in $V$ for the first time. The proof is identical if one defines now $W=\bigcup f^{n_{i}}\left(U_{i}\right)$, because the preimage of one of the sets $f^{n_{i}}\left(U_{i}\right)$ cannot intersect an image of a $U_{j}$.

The open set $\mathcal{U}$ can be diminished again so that $V^{\prime} \backslash g\left(V^{\prime}\right)$ is a fundamental domain whose intersection with $f^{n_{i}}\left(U_{i}\right)$ contains $g^{n_{i}}\left(S_{g} \cap U_{i}\right)$ whenever $U_{i}$ is contained in the basin of $x$ and $g \in \mathcal{U}$. For $i=1,2$, denote by $x_{i}$ the fixed point that the map $g_{i}$ has in $V^{\prime}$.

Definition of the conjugacy $h$ in the neighborhood $V^{\prime}$ of $x_{1}$. It is easy to construct a local conjugacy between $g_{1}$ and $g_{2}$ that is close to the identity, but this local homeomorphism may not preserve critical images. We refer the reader to [IPR1] where a similar construction was done (there, the critical components $U_{i}$ were arbitrarily small and the manifold was twodimensional).

Diminish $\alpha$ again to make it less than the distance between different $f^{n_{i}}\left(U_{i}\right)$ 's. Let $Z_{i}\left(g_{1}\right)$ be the closure of $g_{1}^{n_{i}}\left(U_{i}\right)$ and $Z_{i}\left(g_{2}\right)=\varphi\left(Z_{i}\left(g_{1}\right)\right)$. As $\varphi$ is $\alpha-C^{0}$ close to the identity, it follows that $Z_{i}\left(g_{2}\right)$ contains $g_{2}^{n_{i}}\left(S_{g_{2}} \cap U_{i}\right)$. For each $g=g_{1}, g_{2}$ let $Z_{i}^{\prime}(g)$ be a small neighborhood of $Z_{i}(g)$. The construction 
begins with a homeomorphism

$$
h: V^{\prime} \backslash \bigcup_{i} \bigcup_{n \geq 0} g_{1}^{n}\left(Z_{i}^{\prime}\left(g_{1}\right)\right) \rightarrow V^{\prime} \backslash \bigcup_{i} \bigcup_{n \geq 0} g_{2}^{n}\left(Z_{i}^{\prime}\left(g_{2}\right)\right)
$$

such that $h g_{1}=g_{2} h$ and the $C^{0}$ distance between $h$ and the identity is less than $\varrho$, an arbitrary positive constant to be determined later. Next define $h=\varphi$ in $Z_{i}\left(g_{1}\right)$ and finally extend $h$ to $Z_{i}^{\prime}\left(g_{1}\right) \backslash Z_{i}\left(g_{1}\right)$ so that $h$ is a homeomorphism $\alpha-C^{0}$ close to the identity. To prove that this last extension is possible, note that the boundary of $Z_{i}(g)$ can be taken smooth, and $Z_{i}^{\prime}(g)$ may be taken to be the union of a tubular neighborhood of the boundary of $Z_{i}(g)$ with $Z_{i}(g)$. Note also that the boundary of $f^{n_{i}}\left(U_{i}\right)$ has a finite number of components, so the positive number $\alpha$ can be taken small enough that $\varphi$ identifies components of the boundaries of $Z_{i}\left(g_{1}\right)$ and $Z_{i}\left(g_{2}\right)$ in the same way as $h$ identifies components of the boundary of $Z_{i}^{\prime}\left(g_{1}\right)$ and $Z_{i}^{\prime}\left(g_{2}\right)$. It follows that the problem of constructing this last extension is reduced to showing that a $C^{1}$ map that is $C^{0}$ close to the identity on an embedded manifold, can be extended to a homeomorphism that coincides with the identity outside a tubular neighborhood of it. Once $h$ is defined in the fundamental domain, one can extend it dynamically to the whole $V^{\prime}$.

Definition of $h$ in the basin $B_{g_{1}}$. This part is subdivided into two steps. The first is extending $h$ to the complement in $B_{g_{1}}$ of the union of the preimages of $\bigcup_{i} Z_{i}\left(g_{1}\right)$. First extend $h$ to the first preimage of $V^{\prime}$. For $g=g_{1}, g_{2}$ let

$$
V^{1}(g)=g^{-1}\left(V^{\prime} \backslash \bigcup Z_{i}(g)\right)
$$

Note that $h g_{1}$ is a finite-to-one covering map from each component of $V^{1}\left(g_{1}\right)$ to a component of $V^{\prime} \backslash \bigcup Z_{i}^{\prime}\left(g_{2}\right)$. The map $g_{2}$ is a covering map from each component of $V^{1}\left(g_{2}\right)$ to a component of $V^{\prime} \backslash \bigcup Z_{i}\left(g_{2}\right)$. The domains of these covering maps are homeomorphic and there exists an obvious isomorphism between the first homotopy groups associated. The actions of the corresponding coverings on homotopy groups are equal modulo that isomorphism. From this it follows that there exists a unique lift $\tilde{h}: V^{1}\left(g_{1}\right) \rightarrow V^{1}\left(g_{2}\right)$ such that $h g_{1}=g_{2} \tilde{h}$ and $\tilde{h}\left(x_{1}\right)=x_{2}$. By construction the map $\tilde{h}$ is a homeomorphism that extends $h$.

This procedure can be repeated for further preimages, thus giving an extension of the conjugacy $h$ to a homeomorphism

$$
h: B_{g_{1}} \backslash \bigcup_{n \geq 0} g_{1}^{-n}\left(\bigcup_{i} Z_{i}\left(g_{1}\right)\right) \rightarrow B_{g_{2}} \backslash \bigcup_{n \geq 0} g_{2}^{-n}\left(\bigcup_{i} Z_{i}\left(g_{2}\right)\right) .
$$

The second step is the extension of $h$ to the preimages of $Z_{i}$. The homeomorphism $h$ can be extended in a unique way to a conjugacy defined in the preimages of $g_{1}^{-j}\left(Z_{i}\left(g_{1}\right)\right)$ for all $i$ and $j$ such that $j<n_{i}$, because $g_{1}$ was 
injective there. To define it in $g_{1}^{-n_{i}}\left(Z_{i}\left(g_{1}\right)\right)=U_{i}$ one must use the map $\psi$ of equation (1), to take care of the critical set contained there.

We now claim that $h$ (given by equation (2)) must coincide with $\psi$ in the boundary of $U_{i}$. Note that $f$ is an immersion if restricted to the (smooth) boundary of $U_{i}$. It follows that there exists a number $c>0$ such that two points in the boundary of $U_{i}$ that have the same image under $f$ must be at a distance at least $2 c$. Diminish $\alpha$ again so that $\alpha<c$. If also the neighborhood $\mathcal{U}$ of $f$ is diminished, then the same property holds for every $g$ there. Note also that as the restrictions of both $\psi$ and $h$ to the boundary of $U_{i}$ satisfy the functional equation $\Phi g_{1}=g_{2} \varphi$ (with unknown $\Phi$ ), it follows that $\psi$ and $h$ coincide in a relatively open subset of the boundary of $U_{i}$. But as both maps are $\alpha-C^{0}$ close to the identity, the claim follows. Therefore one can extend $h$ to $U_{i}$ by setting it equal to $\psi$; the remaining extension to the preimages of the sets $U_{i}$ is now obvious.

Extension to the boundary. This part is similar to the proof given in [IPR1]. Fix a neighborhood $U$ of the Julia set of $f$ where $f$ is expanding; say with an adapted metric the differential of $f$ expands in $U$ at a rate $\lambda>1$. By Lemma 6 there exists some positive constant $N$ such that $f^{-n}\left(V^{\prime} \backslash f\left(V^{\prime}\right)\right) \subset U$ for every $n \geq N$. This also holds for every $g \in \mathcal{U}$. Moreover, the constant $\varrho$ given in the definition of $h$ in the fundamental domain can be taken so small that $h$ is $\varepsilon-C^{0}$ close to the identity in $g_{1}^{-N}\left(V^{\prime} \backslash g_{1}\left(V^{\prime}\right)\right)$. Using the expansiveness of $g_{1}$ in $U$ one can show, as in [IPR1, Corollary 3], that $h$ is $\varepsilon-C^{0}$ close to the identity. Then, taking sequences and using the expansiveness of $g_{1}$ in $\Omega^{\prime}\left(g_{1}\right)$, the fact that $h$ extends to the boundary of $B_{g_{1}}$ can be shown as in the above reference. Trivially the extended $h$ is a conjugacy between $g_{1}$ and $g_{2}$. This proves the theorem.

An example. Some examples of perturbations of complex polynomials were shown to be $C^{3}$ structurally stable in [IPR1]. In that case, each component of the set of critical points was a small Jordan curve whose image was disjoint from the interior of the curve. We now show how to construct a stable map in the sphere such that $S_{f}$ is a circle whose image is contained in the component of its complement that contains the fixed attracting point. This map will be a perturbation of the holomorphic map $z \mapsto \varrho z^{2}$. Let $f(x, y)=\varrho\left(x^{2}-y^{2}+\lambda y, 2 x y+\mu x\right)$ be defined in a ball $B_{r}$ of center the origin and radius $r=1 / 2$. If $\varrho, \lambda$ and $\mu$ are small positive numbers, then the origin is an attractor and the set of critical points is a circle contained in $B_{r}$. Moreover, if $\varrho$ is diminished again, then $f\left(S_{f}\right)$ is contained in the bounded component of the complement of $S_{f}$. It can also be seen that the restriction of $f$ to $S_{f}$ is injective, hence $f\left(S_{f}\right)$ is also a topological circle and the origin is contained in the bounded component of its complement. This makes $f$ a $C^{3}$ geometrically stable map. 
To define $f$ in the whole sphere, let it coincide with $z \mapsto z^{2}$ in the annulus $\{z:|z| \in[3 / 4,5 / 4]\}$, and with a map $g$ in the complement of the ball of radius 2 where $1 / g(1 / z)=f(z)$. Then extend $f$ to the whole sphere. The set of critical points of $f$ has two components, each contained in the basin of an attracting fixed point, the origin and $\infty$. The nonwandering set contains an expanding basic piece $\{z:|z|=1\}$. Moreover, the extended map $f$ is still $C^{3}$ geometrically stable. By Theorem $\mathrm{C}$ it follows that $f$ is $C^{3}$ structurally stable.

Final comment. Other examples of maps satisfying the hypothesis of Theorem $\mathrm{C}$ in dimension greater than two can be found in [IPR2] (however, in that case, the maps have no critical points, and the conclusion is that the map is $C^{1}$ structurally stable). To find other examples of structurally stable maps, one would have to admit saddle type basic pieces (or repelling pieces that are not expanding), which represents an additional difficulty, since their unstable manifolds have a wild behavior, as they can have infinitely many intersection points. Przytycki presented the simplest possible example in the last section of $[\mathrm{P}]$. As far as we know, nobody has ever answered his question about the $C^{1}$ structural stability of his example.

Acknowledgements. Thanks are due to the referee for several improvements. The work was partially supported by Conicyt, Uruguay.

\section{References}

[AMS] N. Aoki, K. Moriyasu and N. Sumi, $C^{1}$ maps having hyperbolic periodic points, Fund. Math. 169 (2001), 1-49.

[Ber] P. Berger, Structural stability of attractor-repellor endomorphisms with singularities, Bull. Braz. Math. Soc., to appear.

[F] J. Franks, Necessary conditions for stability of diffeomorphisms, Trans. Amer. Math. Soc. 158 (1971), 301-308.

[GG] M. Golubitsky and V. Guillemin, Stable Mappings and Their Singularities, Grad. Texts Math. 14, Springer, New York, 1973.

[IPR1] J. Iglesias, A. Portela and A. Rovella, Structurally stable perturbations of polynomials in the Riemann sphere, Ann. Inst. H. Poincaré Anal. Non. Linéaire 256 (2008), 1209-1220.

[IPR2] - - - - $C^{1}$ stable maps: examples without saddles, preprint, Premat 2008/106.

[Ma1] R. Mañé, A proof of the $C^{1}$ stability conjecture, Publ. Math. Inst. Hautes Études Sci. 66 (1987), 161-210.

[Ma2] - Axiom A for endomorphisms, in: Lecture Notes in Math. 597, Springer, 1977, 379-388.

[MP] R. Mañé and C. Pugh, Stability of endomorphisms, in: Lecture Notes in Math. 468, Springer, 1975, 175-184.

[P] F. Przytycki, On $\Omega$-stability and structural stability of endomorphisms satisfying Axiom A, Studia Math. 60 (1977), 61-77. 
[R] C. Robinson, Structural stability of $C^{1}$ diffeomorphisms, J. Differential Equations 22 (1976), 28-73.

[S] M. Shub, Endomorphisms of compact differentiable manifols, Amer. J. Math. 91 (1969), 175-199.

J. Iglesias, A. Portela

Facultad de Ingenieria, IMERL

Universidad de La República

Julio Herrera y Reissig 565

Montevideo, Uruguay

E-mail: jorgei@fing.edu.uy aldo@fing.edu.uy
A. Rovella

Centro de Matemática

Facultad de Ciencias

Universidad de La República

Iguá 4225

C.P. 11400, Montevideo, Uruguay

E-mail: leva@cmat.edu.uy

Received 21 April 2008;

in revised form 17 February 2009 